\title{
A Systematic Review of The Systematic Reviews of Post COVID-19 Syndrome
}

\author{
Abdulmohsen Alhumayn, Ibrahim Alsaif, Joud Enabi, and Sharafaldeen Bin Nafisah
}

\begin{abstract}
Background: The declaration of the COVID-19 pandemic triggered a global inquiry into the transmission, mortality, risk factors, and management of the disease. Recently, however, attention has shifted toward its long-term consequences. There is a need for a better understanding of the predictors and symptoms of post-COVID syndrome, to ensure appropriate care for patients recovering from COVID-19 beyond the acute phase.

Methods: We searched PubMed, Google Scholar, Cochrane databases, and available data in the PROSPERO databases. We also explored the reference lists of included articles and any systematic reviews identified therein. We searched the keywords "Post Covid", "Post COVID syndrome", "Post- Covid" and "PostCOVID", from 1963 to July 2021.

Results: Of 8167 articles, 13 were included. The syndrome affects several systems with variable prevalence. Fatigue and sleep disturbance is the most common symptom of acute post-COVID syndrome, observed in more than two-thirds of patients, while a reduction in quality of life and general health status was noted in up to 69\%. Furthermore, a reduced aerobic and diffusion capacity was seen in $38 \%$ of patients up to one month after presumed recovery from infection. Radiologically, in up to $52 \%$ of patients, a ground-glass opacity (GGO) was noted beyond three months post-infection. The incidence of new psychiatric illness increased from as early as $\mathbf{1 4}$ days after infection and up to three or six months. Hearing impairment or loss, whether sensorineural or conductive, was noted in up to $8.3 \%$ of patients, and tinnitus was seen in up to $4.2 \%$.

Conclusion: Overall, given the variability in the manifestation of post-COVID syndrome, a multidisciplinary team is required to better serve these patients. We therefore urge the establishment of such teams, encompassing internal medicine, pulmonology, cardiology, and neurocognitive services.
\end{abstract}

Index Terms-Post COVID, Post COVID complications, Post COVID syndrome

\section{INTRODUCTION}

The declaration of the COVID-19 pandemic triggered immediate inquiry into its transmission, mortality, risk factors, and management. Recently, however, attention has shifted toward its long-term consequences. Several reports highlight the issue of new or persistent symptoms several weeks after presumed recovery [1], the first report thereof probably being in May 2020 [2]. These reports describe an organised syndrome that affects the multisystem in an unorganised fashion, termed "post-COVID-19 syndrome" [3]. The pathophysiology of post-COVID syndrome is believed to be multifactorial. It was proposed that the major contributors to the syndrome

Abdulmohsen Alhumayn, Ibrahim Alsaif, Sharafaldeen Bin Nafisah are with Emergency Department, King Fahd Medical City, e-mail: mohsen.alhumayn@gmail.com, e-mail: ebrahim771@hotmail.com, e-mail: dr.sharafaldeen@yahoo.com (Corresponding author: Abdulmohsen Alhumayn)

Joud Enabi is with Alfaisal University , e-mail: jenabi@alfaisal.edu include, but are not limited to, microvascular ischaemia and injury, immobility, and metabolic alterations during the critical phase of infection [4]. It was also proposed that the syndrome be classified according to the timing of symptoms, degree of recovery, and complications [5]. The duration of symptoms prompted further classification, with symptoms of less than three months but more than five weeks referred to as acute post-COVID symptoms; more than three months but less than 24 weeks termed long post-COVID symptoms; and symptoms beyond 24 weeks referred to as persistent post-COVID symptoms [6-8].

A proper understanding of this syndrome and its predictors is essential to develop an infrastructure capable of caring for those patients recovering from COVID-19 infection beyond the acute phase. It came to our attention that although the literature includes several systematic reviews concerning each system, the reporting of the symptoms of each system still lacks systematic inquiry. This systematic review therefore aims to explore the symptoms of post-COVID-19 and the predictors for its development.

\section{METHODS}

Review question: What are the manifestations and the predictors of post-COVID syndrome?

Study Design: A systematic review of the systematic reviews of post-COVID syndrome.

Search Strategy: We searched PubMed, Google Scholar, Cochrane databases, and available data in the PROSPERO databases. We also explored the reference lists of included articles and any systematic reviews identified therein. Keywords searched were "Post Covid", "Post COVID syndrome", "PostCovid", and "PostCOVID", from 1963 to July 2021.

Selection Criteria: Our inclusion criteria were all systematic reviews of post-COVID syndrome articles that explored patients' symptomatology, irrespective of the syndrome's classification. We excluded non-English articles that lacked translation, and articles that were non-systematic reviews.

Data Extraction and Management: We screened titles and abstracts, extracted published articles, and retrieved data to analyse this systematic review. The studies' eligibility for inclusion was examined by the authors separately; a meeting was then held in case of any disagreement regarding a study's inclusion. The data was extracted on the basis of various factors, including the study's time frame, patient population, investigations, interventions, and outcomes.

We used the Critical Appraisal Skills Programme (CASP) to assess the risk of bias for systematic review [9]. The analysis 
was recorded following the Preferred Reporting Items for a Review and Meta-analysis of Individual Participant Data [10].

Data Synthesis and Summary Measures: We focused on including the descriptive measures of the various signs and symptoms among Post-COVID syndrome patients in each study, using the upper confidence interval limit. We also explored the predictors for the syndrome's development.

\section{Funding RESOURCE:}

No funds, grants, or support were received.

\section{ApProval:}

This study was approved by the research committee, with IRB log number IRB00010471.

\section{RESULTS}

Of 8167 articles, 13 were included, as illustrated in the Prisma chart, Figure 1. The number of participants within each review and the number of articles is illustrated in Table 1, while Table 2 illustrates the risk of bias in those articles.

\section{General health:}

Fatigue is the second-most common symptom of acute postCOVID syndrome, occurring in up to $73 \%$ of patients [11-13], secondary to sleep disturbance in $85 \%$ [11]. While fatigue is still prevalent in chronic post-COVID syndrome, dyspnoea is also prevalent in up to $64 \%$ of patients [11-13]. Nonetheless, fatigue was attributed to many factors, including respiratory muscle weakness, post-viral fatigue syndromes, and possible general deconditioning [12]. Thus, the symptomatology may suggest overlaps with clinical presentation of Myalgic Encephalomyelitis/Chronic Fatigue Syndrome (ME/CFS) [14]. However, any association or causation between post-COVID and those syndromes is yet to be determined, and thus far the similarity has only been suggested [14].

On the other hand, a reduction in quality of life and general health status was noted in up to $69 \%$ of patients from 14 days to three months post-infection [15]. In addition, there was a decline in the performance of activities of daily living (ADL) after COVID-19 infection, regardless of the scale applied [16]. Other non-specific symptoms that affect general health included arthralgia in up to $24 \%$ of patients, neuralgia in $38 \%$, alopecia in $34 \%$, and some had hyperhidrosis $[13,17,18]$.

\section{Respiratory system:}

A reduction in aerobic and diffusion capacity was observed in $38 \%$ of patients within one month after infection. Patients have persistent dyspnoea, cough and chest pain [19]. However, other explanations for the dyspnoea include the development of pulmonary fibrosis, dysfunctional breathing and respiratory muscle weakness that may persist up to eight weeks from recovery $[11,12]$. Residual signs of fibrosis, in particular, were responsible for exercise-induced desaturation [12].

Nevertheless, altered diffusion capacity is the most common pathology, seen in up to $55 \%$ of patients, followed by a restrictive pattern in $17 \%$ and an obstructive pattern in $9 \%$ $[12,19]$. It should be noted that $55 \%$ of patients will have an abnormal pulmonary function tests (PFT) [12]. However, there is an improvement in lung mechanics over time [19].
It should also be asserted that abnormal lung function and structural changes were seen up to six months post-infection [20].

Radiologically, in up to $52 \%$ of patients there is a groundglass opacity (GGO) beyond three months post-infection [12]. During this period, up to $52 \%$ of patients' CTs will reveal interstitial thickening or interlobular septal thickening [12]. In the same period, fibrous stripes were also common and seen in up to $44 \%$ of patients, with a similar percentage for bronchiectasis, in $43 \%$ [12]. Fibrotic-like changes, in particular, were seen up to 6 months post-infection, in up to $35 \%$ of patients [20]. Consolidation was less common, seen in only $11 \%$ [12]. Pleural thickening and bronchovascular bundle distortion were also seen, more commonly than consolation [12]. Autopsies of COVID-19 patients revealed varying degrees of destruction of alveolar structure, as well as pulmonary interstitial fibrosis [19].

\section{Cardiovascular and haematological system:}

Myocardial inflammation was common, as noted in $78 \%$ [11]. This figure varies, however; some studies revealed a lower percentage of $26 \%$ [15]. Acute findings included nonspecific interstitial oedema, with or without myocarditis [21]. However, such findings were not the cause of death in most patients, while hypertrophy or cardiac dilation was noted in the autopsies of those who did not survive [12]. Myocardial fibrosis was more common than myocardial infarction, venous or intracardiac thrombosis, amyloidosis, and inflammatory infiltrate not meeting the diagnosis of myocarditis. However, it was difficult to determine whether these symptoms were acute or overlapping with chronic pathology [12]. Hence, myocardial fibrosis might be the end result of any of the aforementioned cardiac injuries [12].

Symptomatically, chest pain occurred in up to $35 \%$ of patients. This may be a result of the abovementioned causes, or may be related to myocardial ischaemia, thrombosis, myocarditis, perimyocarditis or pericarditis [11,13]. The latter three pathologies appeared as late as three months after onset of symptoms of infection [15]. Even with apparently recovered cardiac function, there might still be a risk of coronary artery disease, atrial fibrillation or ventricular arrhythmias due to myocardial injury [15].

\section{Neurological system and mental health:}

Headache beyond the infection period occurred in up to $78 \%$ of patients $[11,13,17]$. Other symptoms included sensorimotor disturbances, dizziness and vertigo [13,22].

The incidence of new psychiatric illness increased from as early as 14 days and up to three or six months post-infection $[15,22]$. Psychiatric conditions entailed anxiety in $26.8 \%$, depression in $33 \%$, post-traumatic stress disorder in $24.1 \%$, insomnia and dementia, compared with an unaffected control group $[11,15,22]$. Cognitive impairment was observed in up to $35.7 \%$, manifesting as inattention and impaired short-term memory, and was observed up to six months post infection [20]. Moreover, persistent neurological symptoms were noted in up to $55 \%$ of patients after three months [15]. On the other hand, the infection itself might trigger neurodegenerative diseases such as multiple sclerosis, Parkinson's disease, and narcolepsy in the long term [15]. 


\section{Identification of studies via databases and registers}

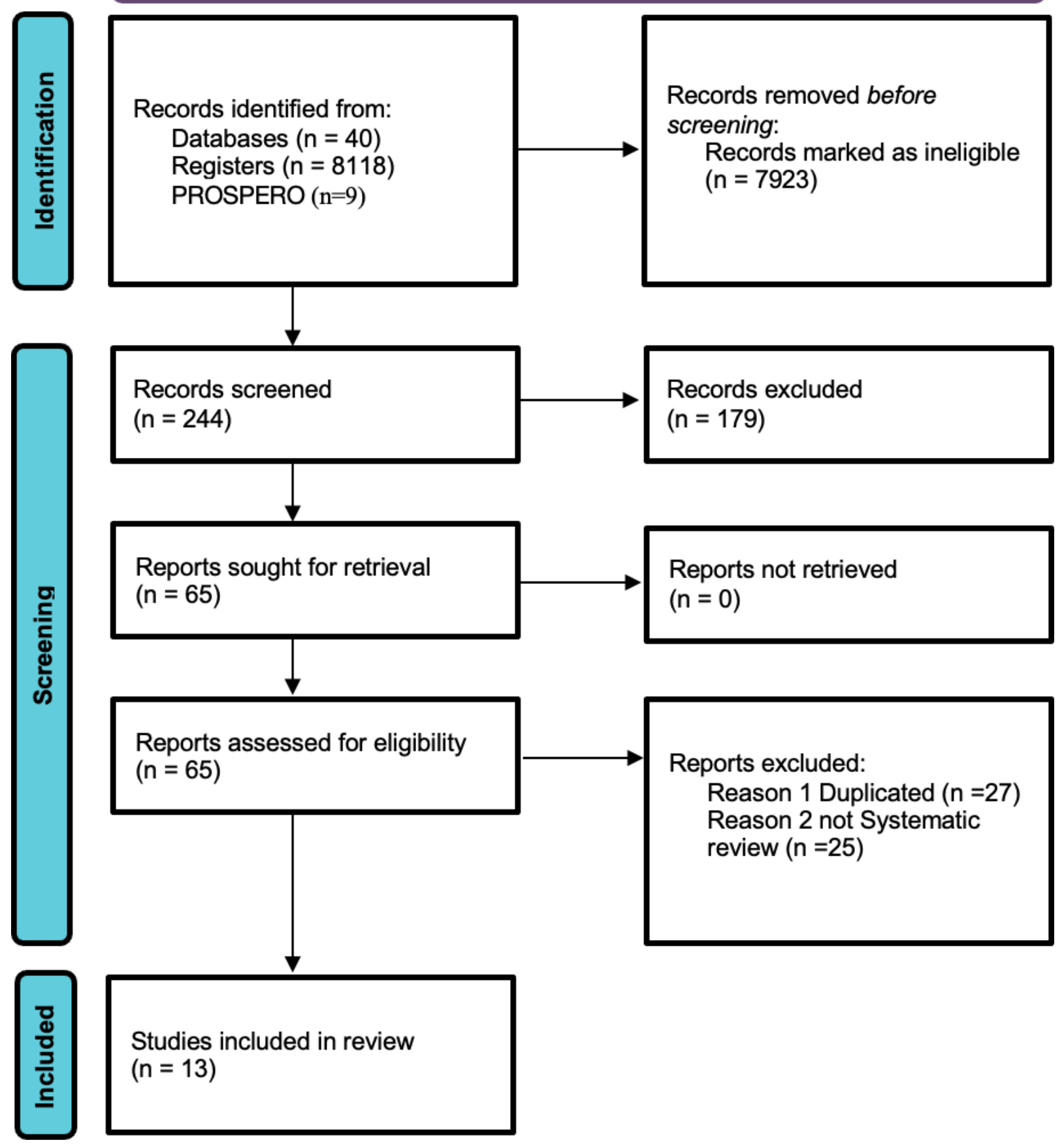

Fig. 1. Prisma chart depicting the identification, screening and inclusion of studies 
TABLE I

NUMBER OF STUDIES REFERENCED AND NUMBER OF PARTICIPANTS WITHIN EACH REVIEW

\begin{tabular}{|c|c|c|c|}
\hline Review & $\begin{array}{l}\text { No. of participants } \\
\text { within review }\end{array}$ & $\begin{array}{l}\text { No. of studies referenced } \\
\text { within review }\end{array}$ & Studies \\
\hline Activities of daily living & 1465 & 9 & $\begin{array}{l}\text { Pizarro-Pennarolli et } \\
\text { al. (2021) }\end{array}$ \\
\hline Cardiovascular, respiratory, neurological and mental health & 12974 & 43 & Iqbal et al. (2021) \\
\hline $\begin{array}{l}\text { Cardiovascular, respiratory, neurological, mental health, endocrine, } \\
\text { haematological and ENT }\end{array}$ & 3520 & 31 & Willi et al. (2021) \\
\hline $\begin{array}{l}\text { Cardiovascular, respiratory, neurological, mental health, endocrine, } \\
\text { haematological and ENT }\end{array}$ & 364 & 35 & $\begin{array}{l}\text { Hoshijima et al. } \\
(2021)\end{array}$ \\
\hline $\begin{array}{l}\text { Cardiovascular, respiratory, neurological, mental health, endocrine, } \\
\text { haematological and ENT }\end{array}$ & 47910 & 15 & $\begin{array}{l}\text { Lopez-Leon et al. } \\
(2021)\end{array}$ \\
\hline ENT & 1874 & 56 & $\begin{array}{l}\text { Almufarrij and } \\
\text { Munro (2021) }\end{array}$ \\
\hline Neurological and mental health & 18917 & 51 & $\begin{array}{l}\text { Badenoch et al. } \\
(2021)\end{array}$ \\
\hline $\begin{array}{l}\text { Cardiovascular, respiratory, neurological, mental health, endocrine, } \\
\text { haematological and ENT }\end{array}$ & 54910 & 21 & Malik et al. (2021) \\
\hline Respiratory & 380 & 6 & $\begin{array}{l}\text { Torres-Castro et al. } \\
(2020)\end{array}$ \\
\hline
\end{tabular}

TABLE II

The Critical Appraisal Skills Programme (CASP) Checklist For Included systematic Reviews

\begin{tabular}{|c|c|c|c|c|c|c|c|c|c|c|c|}
\hline Lead author & Q1 & Q2 & Q3 & Q4 & Q5 & Q6 & Q7 & Q8 & Q9 & Q10 & Fully achieved \\
\hline Pizarro-Pennarolli et al. (2021) & $\mathrm{Y}$ & $\mathrm{Y}$ & $\mathrm{Y}$ & $\mathrm{Y}$ & $\mathrm{Y}$ & $\mathrm{P}$ & $\mathrm{N}$ & Y & $\mathrm{Y}$ & $\mathrm{Y}$ & 8 \\
\hline Iqbal et al. (2021) & $\mathrm{Y}$ & $\mathrm{Y}$ & $\mathrm{P}$ & $\mathrm{Y}$ & $\mathrm{Y}$ & Y & $\mathrm{Y}$ & $\mathrm{Y}$ & $\mathrm{Y}$ & $\mathrm{Y}$ & 9 \\
\hline Roshdy et al. (2021) & Y & $\mathrm{Y}$ & Y & $\mathrm{P}$ & $\mathrm{Y}$ & Y & $\mathrm{N}$ & $\mathrm{Y}$ & Y & $\mathrm{Y}$ & 8 \\
\hline Willi et al. (2021) & $\mathrm{Y}$ & $\mathrm{Y}$ & Y & $\mathrm{Y}$ & $\mathrm{Y}$ & Y & $\mathrm{N}$ & $\mathrm{Y}$ & Y & $\mathrm{Y}$ & 9 \\
\hline Hoshijima et al. (2021) & $\mathrm{Y}$ & $\mathrm{Y}$ & $\mathrm{P}$ & $\mathrm{Y}$ & $\mathrm{Y}$ & Y & $\mathrm{Y}$ & $\mathrm{Y}$ & Y & $\mathrm{Y}$ & 9 \\
\hline Wong and Weitzer (2021) & $\mathrm{Y}$ & $\mathrm{Y}$ & $\mathrm{P}$ & $\mathrm{Y}$ & $\mathrm{Y}$ & Y & $\mathrm{N}$ & $\mathrm{Y}$ & Y & $\mathrm{Y}$ & 8 \\
\hline Lopez-Leon et al. (2021) & Y & $\mathrm{Y}$ & $\mathrm{P}$ & $\mathrm{Y}$ & $\mathrm{P}$ & Y & $\mathrm{P}$ & $\mathrm{Y}$ & $\mathrm{Y}$ & $\mathrm{Y}$ & 7 \\
\hline Almufarrij and Munro (2021) & $\mathrm{Y}$ & $\mathrm{Y}$ & $\mathrm{Y}$ & $\mathrm{Y}$ & $\mathrm{Y}$ & $\mathrm{P}$ & $\mathrm{P}$ & $\mathrm{Y}$ & $\mathrm{Y}$ & $\mathrm{Y}$ & 8 \\
\hline Malik et al. (2021) & $\mathrm{Y}$ & $\mathrm{Y}$ & $\mathrm{N}$ & $\mathrm{Y}$ & $\mathrm{Y}$ & $\mathrm{P}$ & $\mathrm{N}$ & $\mathrm{Y}$ & $\mathrm{Y}$ & $\mathrm{Y}$ & 7 \\
\hline Sanchez-Ramirez et al. (2021) & Y & $\mathrm{Y}$ & Y & $\mathrm{Y}$ & Y & $\mathrm{P}$ & $\mathrm{Y}$ & $\mathrm{Y}$ & Y & $\mathrm{Y}$ & 9 \\
\hline Salamanna et al. (2021) & Y & $\mathrm{Y}$ & Y & $\mathrm{Y}$ & Y & $\mathrm{N}$ & $\mathrm{N}$ & $\mathrm{Y}$ & $\mathrm{N}$ & $\mathrm{Y}$ & 7 \\
\hline Torres-Castro et al. (2020) & $\mathrm{Y}$ & $\mathrm{Y}$ & $\mathrm{Y}$ & $\mathrm{Y}$ & $\mathrm{Y}$ & $\mathrm{Y}$ & $\mathrm{P}$ & $\mathrm{Y}$ & $\mathrm{N}$ & $\mathrm{Y}$ & 8 \\
\hline
\end{tabular}

Y: Yes; N: No; P: Partially

\section{Otorhinolaryngological system:}

Hearing impairment/loss, whether sensorineural or conductive, was noted in up to $8.3 \%$ of patients $[20,23]$. The extent of impartment varies, but it appears to occur at high frequencies [23]. Tinnitus was also noted in up to $4.2 \%$, occurring intermittently or continuously [20]. Such symptoms may have been present before the infection, however, and only exacerbated later on [23]. Non-specific vertigo, otalgia, otitis media, and other symptoms related to hypersensitivity and phonophobia were also noted [20,23]. Anosmia also occurred in post-COVID syndrome in up to $27 \%$ of patients $[11,13]$. The olfactory dysfunction observed in these patients follows a similar mechanism to that of the post-viral olfactory dysfunction seen in other common viral infections, such as rhinovirus [15]. The olfactory and gustatory dysfunctions did not improve after one month, in $28 \%$ and $20 \%$ of patients retrospectively. The former persisted in up to $14.3 \%$ after the second month, and some cases remained up to six months [20]. Ageusia was also reported in up to $28 \%$ of patients [11].

\section{Endocrinological and reproductive system:}

Endocrine manifestations included subacute thyroiditis, hypothalamic-pituitary-thyroid axis dysfunction, diabetes secondary to pancreatic damage, and testicular dysfunction with associated infertility [15].

\section{Predictors for the development of post-COVID syndrome:}

The worse the initial presentation, the more likely it is that the patient will report functional impairment later on [11]. Moreover, the higher the symptom load, the more likely it will persist, and the more likely it will subsequently develop persistent fatigue associated with long term impairment [11]. However, some argued against the association of disease severity with a higher prevalence of fatigue [12]. The need for hospitalisation and the 40-49-year age segment were also predictors for the development of post-COVID syndrome [11]. Higher mortality was observed in the short term among patients with a low baseline score on the Barthel Index for Activities of Daily Living (ADL), i.e., decreased functional status and dependency. This was seen among older age groups and patients with a complicated hospital stay, including the need for mechanical ventilation and ICU care [16]. 
The severity of the altered diffusion capacity is associated with the development of pulmonary fibrosis. Moreover, high inflammatory markers signify severe disease [19]. Those with severe disease had persistent lung abnormalities on advanced imaging [12]. Increased oxygen requirement is associated with ground-glass opacification/opacity and the diffusing capacity of the lungs for carbon monoxide (DLCO) [12]. Symptomatically, residual abnormalities in CXR correlated with persisting respiratory symptoms up to six months post-infection [20].

In addition, moderate to severe COVID is associated with neurocognitive impairment in up to $37 \%$ of patients for a period of up to six months [20]. The prevalence of neuralgia appears to increase with time [13]. Moreover, non-hospitalised patients were more likely to develop anxiety [22].

\section{DISCUSSION}

At the time of this writing, this is the first comprehensive review of post-COVID syndrome. This review uncovered a broad range of symptoms that persist beyond assumed recovery from COVID infection. It is therefore prudent to explore the prevalence of such symptoms and their onset, in order to reduce the frequency of patient visits to the ED and to plan for subsequent care after discharge. Due to the variation in symptoms and the multiple systems affected post-COVID, it is also prudent to establish an interdisciplinary team to follows every patient with this syndrome.

The symptomatology of post-COVID syndrome varies in the literature; however, fatigue, dyspnoea and sleep disturbance are common in the early phase. Fatigue was indeed noted after several infections, including influenza A (H1N1) virus, SARS-CoV, Ebolavirus and West Nile virus [15]. Although fatigability might be multifactorial here, some advocate the implication of muscular atrophy and the use of steroids [24]. Dyspnoea was related to altered diffusion capacity, acutely, or the development of pulmonary fibrosis or respiratory muscle weakness. The chronic lung changes that occurred during the infection were responsible for the dyspnoea that occurred after resolution of the infection. Lung damage from other Coronavirus strains also manifested with dyspnoea [25]. It is assuring to note that the lung mechanics improve with time; however, it may require six months for complete improvement [20]. In this review, we noted that up to one-third of patients developed pulmonary fibrosis. Therefore, a follow up with advanced imaging is required in those presenting with the respiratory symptoms.

Several myocardial changes were also noted in relation to post-COVID syndrome. Myocarditis was also seen, but was not severe enough to be a cause of death [12]. Chest pain should be worked up for myocardial ischaemia, thrombosis, myocarditis, perimyocarditis and pericarditis $[11,13]$. Those with chest tightness will also require investigation for a secondary chest infection or stress-induced cardiomyopathy [26]. Because myocarditis was not common, it should not be among the top differential diagnoses as a cause of cardiovascular deterioration [21]. Arrhythmias should also not be overlooked [15]. Moreover, due to the variation in post-Covid cardiovascular findings, MRI is warranted, whether on an acute emergency visit or as an inpatient.
Headache is known to be a common presentation of Covid19 infection, and also in the post-infection period. Although $78 \%$ of patients developed a headache as part of their syndrome, it should trigger investigating for cerebral venous thrombosis as well, given the thrombogenicity of the virus [27].

New-onset psychiatric illness was not uncommon, and mental health assessment should be included in the patient's follow-up. While more than half the patients had a persistent neurological deficit after three months, we noted that cognitive impairment was seen up to six months $[15,20]$; this mandates the integration of a neurocognitive service in the follow-up period.

Probably the least common manifestation of this syndrome is the otorhinolaryngological symptoms and the endocrinological and reproductive symptoms and, given the inconsistent findings, a follow-up should not be standardised.

Overall deconditioning should be expected, mandating frequent outpatient visits, and more involvement could be needed from the patient's family or from social services. Furthermore, decreased functional status and dependency should be assessed regularly, as these were associated with short-term mortality [16].

The association of the initial presentation and higher symptom load with the development of this syndrome is a nonmodifiable factor. With widespread vaccination, however, this may change. Nevertheless, patients with a severe presentation, especially with high inflammatory markers, should have regular and more frequent follow-ups given their propensity for persistent lung abnormalities, neurocognitive impairment and fatigue, all of which lead to a decline in general health status. Whether the reduction in quality of life and general health status is related to dyspnoea or to fatigue and joint pain is yet to be determined. However, we believe it stems from multifactorial causes, including organic and psychological factors.

\section{CONCLUSION}

Overall, given the variability in the manifestation of postCOVID syndrome, a multidisciplinary team is needed to better serve these patients. We therefore urge the establishment of such teams, encompassing internal medicine, pulmonology, cardiology, and neurocognitive services.

\section{REFERENCES}

[1] Al-Jahdhami I, Al-Naamani K, Al-Mawali A. The Postacute COVID-19 Syndrome (Long COVID). Oman Med J. 2021 Jan 26;36(1):e220. doi: 10.5001/omj.2021.91. PMID: 33537155 ; PMCID: PMC7838343.

[2] Report: What Does COVID-19 Recovery Actually Look Like? Patient Led Research Collaborative, https://patientresearchcovid19.com/research/report-

1/\#Summary, 6 Dec. 2020, patientresearchcovid19.com/research/report-1/\#Summary.

[3] Carfi A, Bernabei R, Landi F \& Gemelli Against COVID19 Post-Acute Care Study Group. Persistent symptoms in patients after acute COVID-19. J. Am. Med. Assoc. 324, 603605 (2020).

[4] Inoue $\mathrm{S}$ et al. Post-intensive care syndrome: its pathophysiology, prevention, and future directions. Acute Med. Surg. 6, 233-246 (2019). 
[5] Maltezou HC, Pavli A, Tsakris A. Post-COVID Syndrome: An Insight on Its Pathogenesis. Vaccines (Basel). 2021 May 12;9(5):497. doi: 10.3390/vaccines9050497. PMID: 34066007; PMCID: PMC8151752.

[6] Fernandez-de-Las-Penas C, Palacios-Cena D, GomezMayordomo V, Cuadrado ML, Florencio LL. Defining postCOVID symptoms (post-acute COVID, long COVID, persistent post-COVID): An integrative classification. Int. J. Environ. Res. Public Health. 2021;18:2621. doi: 10.3390/ijerph18052621.

[7] Van Kampen JJA et al. Duration and key determinants of infectious virus shedding in hospitalised patients with coronavirus disease-2019 (COVID-19). Nat. Commun. 12, 267 (2021).

[8] Greenhalgh T, Knight M, A'Court C, Buxton M, Husain L. Management of post-acute COVID-19 in primary care. Brit. Med. J. 370, m3026 (2020).

[9] Brice, Ruth. CASP CHECKLISTS. CASP - Critical Appraisal Skills Programme, 20 Nov. 2020, casp-uk.net/casptools-checklists.

[10] Page MJ et al. The PRISMA 2020 Statement: An Updated Guideline for Reporting Systematic Reviews. BMJ, 2021, p. n71. Crossref, doi:10.1136/bmj.n71.

[11] Iqbal FM et al. Characteristics and Predictors of Acute and Chronic Post-COVID Syndrome: A Systematic Review and Meta-Analysis. EClinicalMedicine, vol. 36, 2021, p. 100899. Crossref, doi:10.1016/j.eclinm.2021.100899.

[12] Sanchez-Ramirez DC et al. Long-Term Impact of COVID-19: A Systematic Review of the Literature and MetaAnalysis. Biomedicines, vol. 9, no. 8, 2021, p. 900. Crossref, doi:10.3390/biomedicines9080900.

[13] Hoshijima $\mathrm{H}$ et al. Incidence of Long-Term Post-Acute Sequelae of SARS-CoV-2 Infection Related to Pain and Other Symptoms: A Living Systematic Review and Meta-Analysis. Medrxiv, 2021. Crossref, doi:10.1101/2021.04.08.21255109.

[14] Wong TL, Weitzer DJ. Long COVID and Myalgic Encephalomyelitis/Chronic Fatigue Syndrome (ME/CFS) - A Systemic Review and Comparison of Clinical Presentation and Symptomatology. Medicina, vol. 57, no. 5, 2021, p. 418. Crossref, doi:10.3390/medicina57050418.

[15] Willi $\mathrm{S}$ et al. COVID-19 Sequelae in Adults Aged Less than 50 Years: A Systematic Review. Travel Medicine and Infectious Disease, vol. 40, 2021, p. 101995. Crossref, doi:10.1016/j.tmaid.2021.101995.

[16] Pizarro-Pennarolli C, Sánchez-Rojas C, Torres-Castro R, Vera-Uribe R, Sanchez-Ramirez DC, Vasconcello-Castillo L, Solís-Navarro L, Rivera-Lillo G. Assessment of activities of daily living in patients post COVID-19: a systematic review. PeerJ. 2021 Apr 6;9:e11026. doi: 10.7717/peerj.11026. PMID: 33868804; PMCID: PMC8034364.

[17] Lopez-Leon S et al. More than 50 Long-Term Effects of COVID-19: A Systematic Review and Meta-Analysis. Scientific Reports, vol. 11, no. 1, 2021. Crossref, doi:10.1038/s41598-021-95565-8.

[18] Malik J et al. Post-Acute COVID-19 Syndrome and Its Prolonged Effects: An Updated Systematic Review. Medrxiv, 2021. Crossref, doi:10.1101/2021.05.09.21256911.

[19] Torres-Castro R et al. Respiratory Function in Patients Post-Infection by COVID-19: A Systematic Review and MetaAnalysis. Pulmonology, 2020. Crossref, doi:10.1016/j.pulmoe.2020.10.013.

[20] Salamanna F et al. Post-COVID-19 Syndrome: The Persistent Symptoms at the Post-Viral Stage of the Disease. A
Systematic Review of the Current Data. Frontiers in Medicine, vol. 8, 2021. Crossref, doi:10.3389/fmed.2021.653516.

[21] Roshdy A, Zaher S, Fayed H, Coghlan JG. COVID-19 and the Heart: A Systematic Review of Cardiac Autopsies. Front Cardiovasc Med. 2021 Jan 28;7:626975. doi: 10.3389/fcvm.2020.626975. PMID: 33585586; PMCID: PMC7876291.

[22] Badenoch JB et al. Persistent Neuropsychiatric Symptoms after COVID-19: A Systematic Review and Meta-Analysis. Medrxiv, 2021. Crossref, doi:10.1101/2021.04.30.21256413.

[23] Almufarrij I, Munro KJ. One Year on: An Updated Systematic Review of SARS-CoV-2, COVID-19 and AudioVestibular Symptoms. International Journal of Audiology, 2021, pp. 1-11. Crossref, doi:10.1080/14992027.2021.1896793.

[24] Disser NP, De Micheli AJ, Schonk MM, et al. Musculoskeletal consequences of COVID- 19. J Bone Joint Surg Am. 2020;102(14):1197-1204. doi: 10.2106/jbjs.20.00847 [25] Helding L, Carroll TL, Nix J, Johns MM, LeBorgne WD, Meyer D. COVID-19 after effects: concerns for singers. J Voice 2020. https://doi.org/10.1016/j. jvoice.2020.07.032

[26] Siripanthong B, Nazarian S, Muser D, Deo R, Santangeli P, Khanji MY, Cooper LT Jr, Chahal CAA. Recognising COVID-19-related myocarditis: The possible pathophysiology and proposed guideline for diagnosis and management. Heart Rhythm. $2020 \quad$ Sep;17(9):1463-1471. doi: 10.1016/j.hrthm.2020.05.001. Epub 2020 May 5. PMID: 32387246; PMCID: PMC7199677.

[27] Sugiyama Y, Tsuchiya T, Tanaka R, Ouchi A, Motoyama A, Takamoto T, Hara N, Yanagawa Y. Cerebral venous thrombosis in COVID-19-associated coagulopathy: A case report. $J$ Clin Neurosci. 2020 Sep;79:30-32. doi: 10.1016/j.jocn.2020.07.038. Epub 2020 Jul 23. PMID: 33070913 ; PMCID: PMC7377797. 